Forschende

Komplementärmedizin und

Klassische Naturheilkunde

\title{
Was mir Kopfschmerzen macht!
}

Es ist erst ein paar Monate her, dass E. Ernst in dieser Zeitschrift ein Editorial unter dem Titel «Publish and Perish» veröffentlicht hat. Einer seiner Kernsätze lautete: «Einer der besten Gründe zu publizieren ist, die zukünftige medizinische Versorgung zu verbessern.» [1] Wir alle, die wir dieses Editorial gelesen haben, haben zumindest innerlich zustimmend genickt. Es gehört zu unserem Selbstverständnis, dass die (wissenschaftliche) Publikation eine zentrale Grundlage dafür ist, dass Fortschritt passiert und dass Patienten von diesem Fortschritt profitieren können. Dieses Credo ist letztendlich die Voraussetzung dafür, dass evidenzbasierte Medizin überhaupt möglich ist: Wie Chalmers, Gray und Sheldon betonen, ist es aus dieser Sicht grundsätzlich unethisch, Forschungsergebnisse nicht zu publizieren [2].

Nun kam mir vor einigen Tagen zufällig ein Prospekt ins Haus geflattert. Es handelte sich um eine der üblichen Hochglanzbroschüren, die Pharmafirmen in grosser Zahl zu Werbezwecken unter die Ärzte bringen. Diese spezielle warb für ein homöopathisches Komplexmittel zur Behandlung der Migräne. In knappen Worten und bunten Bildern wurde eine doppelblinde, plazebokontrollierte, randomisierte Studie vorgestellt, die signifikant positive Effekte zugunsten des homöopathischen Verums zeigte.

Homöopathie und Migräne? Das lässt uns stutzen! Fast jeder wissenschaftlich an der Homöopathie Interessierte hat zu diesem Thema ein ungutes Gefühl. Es ist die Geschichte eines Forschungsmisserfolgs, der in der wissenschaftlichen Landschaft zur Homöopathie tiefe Narben hinterlassen hat. Inspiriert von dramatisch guten Ergebnissen Ende der 80er Jahre hatten mehrere Forschergruppen die Migräne bzw. Spannungskopfschmerzen als das klinische Modell gewählt, an dem die Wirksamkeit der Homöopathie (insbesondere der Einzelmittelhomöopathie) exemplarisch bewiesen werden sollte. Herausgekommen sind drei randomisierte klinische Studien, die neben der Tatsache, dass sie alle hohen Qualitätsansprü- chen genügen, eines gemeinsam haben: Sie konnten keine Überlegenheit des homöopathischen Verums über Plazebo nachweisen. Allein die Literatur der Mutmassungen, wie es zu diesen Negativergebnissen kommen konnte, füllt Aktenordner. Die Erkenntnis blieb dennoch: «Die vorhandenen Studiendaten deuten nicht darauf hin, dass die Homöopathie in der Migräne- oder Kopfschmerzprophylaxe einen über Plazebo hinausgehenden Effekt aufweist». [3]

Und nun dieses: eine klinische Studie zur homöopathischen Migränetherapie mit positivem Ausgang! Ich will an dieser Stelle nicht darüber spekulieren, ob sich die obige Schlussfolgerung dadurch ändert. Mir geht es hier um etwas ganz anderes, nämlich um die Frage, warum diese Studie (soweit ich recherchieren konnte) nie wissenschaftlich publiziert wurde. Soweit die Broschüre eine Beurteilung zulässt, kann es nicht an der Qualität der Studie gelegen haben, Zeitschriften wie die «Forschende Komplementärmedizin und Klassische Naturheilkunde» wären sicherlich gern bereit gewesen, eine entsprechende Publikation vorzunehmen. Meine Vermutung ist eine ganz andere: Für Pharmafirmen aus dem komplementärmedizinischen Sektor ist es letztendlich überhaupt nicht interessant, wissenschaftlich zu veröffentlichen! Nicht die Veröffentlichung zählt, sondern allein das Marketing, insbesondere, wenn die anzusprechende Klientel dem Begriff Wissenschaft eher skeptisch gegenübersteht. Aus Sicht der Pharmafirma dürfte eine Hochglanzbroschüre das therapeutische Verhalten der interessierten Ärzte wesentlich mehr beeinflussen als eine Veröffentlichung in einer Fachzeitschrift. Der Impact der Publikation ist gleich Null. Schliesslich hat auch die bisherige Datenlage nicht dazu geführt, dass Homöopathen oder homöopathisch arbeitende Ärzte ihre Migräneprophylaxe umgestellt haben.

Wir sollten nicht darüber lamentieren, dass das Verhalten der Pharmafirma und des Autors unethisch ist. Vielmehr können wir in dem Vorgang ein Symptom sehen, ein Symptom für ein

\begin{tabular}{ll}
\hline KARGER & @ 2004 S. Karger GmbH, Freiburg \\
$\begin{array}{l}\text { Fax +497614520714 } \\
\begin{array}{l}\text { E-mail Information@Karger.de } \\
\text { www.karger.com }\end{array}\end{array}$ & $\begin{array}{l}\text { Accessible online at: } \\
\text { www.karger.com/fkm }\end{array}$
\end{tabular}

Dipl. Stat. Rainer Lüdtke

Karl und Veronica Carstens-Stiftung

Am Deimelsberg 36

D-45276 Essen

Tel. +49 20156 305-16, Fax -30

E-mail r.luedtke@carstens-stiftung.de 
grundlegendes Problem: Augenscheinlich ist es in der Komplementärmedizin noch nicht gelungen, die Bedeutung der wissenschaftlichen Publikation ins rechte Licht, d.h. in das Bewusstsein der Ärzte, zu rücken. Es gilt nicht, mit dem Finger auf andere zu zeigen und den ersten Stein zu werfen. Vielmehr sollten sich Zeitschriften wie die Forschende Komplementärmedizin Gedanken machen, wie sie Autoren positiv motivieren können, ihre Ergebnisse wissenschaftlich zu veröffentlichen. Wissenschaftliche Zeitschriften müssen für niedergelassene Ärzte lesbarer werden, nur wenigen ist das bisher gelungen (das überragende Bespiel scheint mir das British Medical Journal zu sein). Vor allem in der Darstellung der Ergebnisse können wissenschaftliche Journals viel von den Hochglanzbroschüren lernen.

Im Übrigen glaube ich, dass es eine sehr kurzsichtige Politik ist, Forschungsergebnisse nicht wissenschaftlich $\mathrm{zu}$ publizieren. Vor allem homöopathische Arzneimittel werden nur dann eine Chance haben, irgendwann wieder erstattungsfähig zu werden, wenn es genügend wissenschaftliche Evidenz gibt, dass sie wirksam sind. Das wirtschaftliche Überleben gerade kleiner Pharmahersteller dürfte aber mit diesem Wirksamkeitsnachweis eng verknüpft sein.

R. Lüdtke, Essen

\section{Literatur}

1 Ernst E: Publish and perish. Forsch Komplementärmed Klass Naturheilkd 2003; 10:240-241.

2 Chalmers I, Gray M, Sheldon T: Prospective registration of health care research would help. BMJ 1995;311:262.

3 Ernst E: Homeopathic prophylaxis of headaches and migraine? A systematic review. J Pain Symptom Management 1999;18:353-357. 